\title{
Contents, Vol. 130, 1955
}

\section{Index}

Agarwal, L. P., and R. L. Malhoutra, Conjunctival Smear Cytology in Xerosis 378

and R. P. Saxéna, Role of Diamox in Glaucoma 164

K. S. Jindal, and R. P. Saxéna. Role of Aqueous Humour Cytopathology in Differential

Diagnosis of Iridocyclitis 272

Beheyt, J., vide /. Francois.

Bleeker, G. M., and E. R. Maas, The Penetration of Aureomycin, Terramycin and Chloramphenicol in the Ocular Tissues

fiouman, M. A., vide J. Ten Doesschate.

Cascio, G., Sur la «duplicatio supercilii» 231

Colenbrander, M. C: Scheme for Conversion of Cylindrical Glasses ... 83

- $\quad$ Two Optical Systems of Cylinder Glasses 84

Discussion: Roelofs.

- $\quad$ A New Test Type 219

- How Can Administration of Oxygen Give Rise to Anoxaemia? .... 220Copper, A. C:

Clinical Contribution to the Knowledge of the Course of the

Nerve Fibres in the Retina 81

Discussion: Zeeman, Copper (reply).Dekking, H. M., Arteriovenous Aneurysm of the Retina

with Spontaneous Regression

- Demonstrations 80

Discussion: Rochat, Flieringa, Dekking (reply).

- $\quad$ An Instrument for Measuring Visual Power 225

Discussion: Waardenburg, Mrs. Kok-van Alphen, Dekking (reply).

Doesschate, J. ten, Electrophysiological Measurement of Contrast-Sensitivity

on the Frog Retina 224

Discussion: Bouman, Ten Doesschate (reply).

- $\quad$ and M. P. Lansberg, Glaucoma and Audiogram 66

Discussion: Dekking, Hagedoorn, Ten Doesschate (reply).

- $\quad$ and M. A. Bouman: Photochemical and Nervous Components of Visual

Adaptation 67

Discussion: Jonkers, Ten Doesschate (reply).

Ebethartinger, W., vide II. Schenk.

Focosi, M., and G. C. Guzzinati, The Head Tilting Test in the Study of Vertical

Component Associated with Convergent or Divergent Squint .... 283 Forgoes, J., vide P.

Weinstein.

Forni, S., Un nouveau centreur-marqueur pour la kératoplastie lól

Francois, J., et /. Beheyt, Cataracte par rayons X et cystéamine 397

Frassineti, A., vide V. Rizzini.

Gafner, F., und H. Goldmann, Experimentelle Untersuchungen über den Zu- 
sammenhang von Augendrueksteígerung und Gesichtsfeldschädigung . . 357 Goldmann, H., vide F. Gafner.

Gramberg-Danielsen, P., Die Rehandlung des Glaucoms mit Megaphen . . 403 Guzzinati, G. C, vide M. Focosi.

GgÖГffy, I., Correction of Unilateral Aphakia with Contact Lens 329

Henkes, H. E., Electrorelinogram in Retinal Hypertension and Arteriosclerosis 68

Discussion: Colenbrander, Leffertstra, Dekking, Winkelman. Henkes (reply).Heuvel, J. E. A. van den, Cytology of the Epithelium of the Crystalline Lens . . 79

- $\quad$ Precipitates on the Vitreous 191

Janssens, G. J. A.: The Results of «Shortening» of the Bulbus by «Reefing» of the Sclera in the Year $1953 \quad 227$

Jenny, E, vide F. Rintelen.

Jindal, K. S., vide L. P. Agarwal.

Jonkers, G. H.: The Freeman Near-Vision Unit 218

- $\quad$ The Results of 5 Years Orthoptic Training 69

Discussion: Mrs. Kok-Van Alphen. Koster, Horst, O. Roelofs, Crone, Winkelman, Jonkers

(reply).

Kronfeld, P. C: A New Calibration Scale for Schiøtz, Gradle Schiøtz and McLean

Tonometers 155

Krümmel, H., und L. Rausch: Anomalien des Auges bei der sogenannten In-continentia

pigmenti. Beürag zur Differentialdiagnose und Pathogenese desPseudoglioms gegenüber der

retrolentalen Fihroplasie 31

Landau, J., vide M. Streifler.

Lansberg, M. P., vide J. ten Doesschate.

Leonardi, F., vide P. Niesel.

Lepri, G., Posttraumatic Neoformation in the Iris, with Atypical Aspect and

Constitution $\quad .412$

Maas, E. R., vide G. M. Meeker.

Malbran, E, vide J. Malbran.

Malbran, J., A. L. Norbis et E. Malbran, Ligne de conduite et traitement de la

paralysie du grand oblique 97

Malhoutra, R. L., vide L. P. Agarwal.

Mazanec, A'., vide V. Vejdousky.

Miescher, A., Experimentelle Untersuchungen zur Pathogenese der Sympathi-

schen Ophthalmie 128

Mulock Houwer, A. W,: Demonstration of Pathological Slides 224

Niesel, P., und F. Leonardi, Vergleichende Eichversuche mit dem Angiotono-

meter nach Baurmann und dem Ophthalmodynamometer nach H. K. Müller 295 Norbis, A. L.,

vide /. Malbran. Nordheim, R. W. vvn, «Naphazoline», an Effective Curative in the Treatment of Irido-Cyclitis and Glaucoma 85

Discussion: Dekking, Henkes, van den Heuvel, van der Weyden, von Nordheim (reply).

Oláh,E., Sur Гinsuffisance de la capacité du regard 321

Orbán, T, Beiträge zu den Augenhintergrundsveränderungen hei Sclerosis

multiplex 387

Palíc-Szánto, ()., Primärer und isolierter Lupus erythematodes am Augenlid . 180

Papolczy, ¥., Staroperation hochgradig myopischer Augen 
Pau, H, Das polarisationsoptische Verhalten von Hornhaut und Lederhaut unter verschiedenen Bedingungen $\quad 340$

Peterhans, A., Statistische Betrachtungen an 426 in der Basler Augenklinik behandelten Fallen von Herpes corneae 244

Planten, J. Th.: A Case of Retrolental Fibroplasia Terminating in an Ahlatio

Falciformis 214

- Pseudo-Papilloedema 216

Discussion: Vos, von Winning, Weve, Dekking, Copper, Planten (reply). Raáb, C, vide A.

Sternberg.

Radnót, M., Der intraokulare Druck hei Tumoren der Hypophysengegend . . 209 Rausch, L·.i vide H. Krümmel.

Rintelen, F., und E. Jenny. Über «Diamox»-Therapie beim Glaukom . . 171

Rizzini, V., et A. Frassineti, In cas d'artérite temporale à issue fatale . . 178 Sakic, D, Contribution à la technique de la trepanation de $\Gamma$ os an cours de la

dacryocysto-rhinostomie. (Modification apportée à Гécarteur d'Axenfeld.) . 336 Saxéna, R. P., vide L. P. Ayarwal. Schenk, //., und W. Eberhartinger, Untersuchungen des Augendruckes nach Verabreichung blutdrucksenkender Mittel 312

Sternberg, A., und C. Raáb, Theoretisches und Praktisches über ein neues Mus-

kelschema 53

Straub, W, Experimentelle Infektion der Kaninchen-Hornhaut mit Toxoplasma gondii 200

Streifler, M, and ./. Landau. Electrical Brain Potentials in Retinitis Pigmentosa and Familial Hemeralopia 116

Vejdovsky, V., et Ä. Mazanec, Examen histologique d'un greffon de cornée transparent après la kératoplastie $\quad 344$

Vrabec, Fr, Sur les rapports des fibres nerveuses régénérées de la cornée humaine avec les kératoblastes 24

Weinstein, P., and ,/. Forgoes, Data Concerning the Mechanism of the Eye Tension Lowering Effect of Diamox 159

Weve, H. J. M.: Demonstrations 227

GESELLSCHAFTSBERICHTE - SOCIETY TRANSACTIONS - SOCIÉTÉS

Netherlands Ophthalmological Society.

131st Meeting in Groni'ngen, May 8th and 9th, $1954 \quad 65$

132nd Meeting in Utrecht, December 11th and 12th, 1954213

BUCHBESPRECHUNGEN - BOOK REVIEWS -- LIVRES NOUVEAUX

$94,156.229,353$

N. NECROLOGIA 230

VARIA 94

157

230 